TITLE:

\title{
Confinement effect of laser ablation plume in liquids probed by self- absorption of C-2 Swan band emission
}

\author{
$\operatorname{AUTHOR}(\mathrm{S}):$
}

Sakka, T; Saito, K; Ogata, YH

\section{CITATION:}

Sakka, T ... [et al]. Confinement effect of laser ablation plume in liquids probed by selfabsorption of C-2 Swan band emission. JOURNAL OF APPLIED PHYSICS 2005, 97(1): 014902.

\section{ISSUE DATE:}

2005-01-01

URL:

http://hdl.handle.net/2433/50404

\section{RIGHT:}

Copyright 2005 American Institute of Physics. This article may be downloaded for personal use only. Any other use requires prior permission of the author and the American Institute of Physics. 


\title{
Confinement effect of laser ablation plume in liquids probed by self-absorption of $\mathrm{C}_{2}$ Swan band emission
}

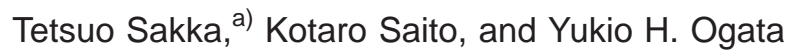 \\ Institute of Advanced Energy, Kyoto University, Uji, Kyoto 611-0011, Japan
}

(Received 12 July 2004; accepted 8 October 2004; published online 10 December 2004)

\begin{abstract}
The $(0,0)$ Swan band of the $\mathrm{C}_{2}$ molecules in a laser ablation plume produced on the surface of graphite target submerged in water was used as a probe to estimate the density of $\mathrm{C}_{2}$ molecules in the plume. Observed emission spectra were reproduced excellently by introducing a self-absorption parameter to the theoretical spectral profile expected by a rotational population distribution at a certain temperature. The optical density of the ablation plume as a function of time was determined as a best-fit parameter by the quantitative fitting of the whole spectral profile. The results show high optical densities for the laser ablation plume in water compared with that in air. It is related to the plume confinement or the expansion, which are the important phenomena influencing the characteristics of laser ablation plumes in liquids. () 2005 American Institute of Physics.
\end{abstract}

[DOI: $10.1063 / 1.1828214]$

\section{INTRODUCTION}

Laser ablation of solid targets submerged in liquids is attracting increasing attention, because of its potential applications, such as nanocluster formation directly in liquid, and the metastable phase formation on the target surfaces. ${ }^{1-6}$ The characterization of the laser ablation plume in liquids, especially the confinement effect, is an important subject to clarify the formation mechanism of such materials. ${ }^{7-15}$

It has been suggested that the ablation plume in liquids is confined to a small region due to the presence of the liquid. As a result, the plume shows very high pressure, as well as high density of the ablated species. Such confinement has been suggested by the theory and measurements of the shock wave which is generated by the pulsed laser irradiation to water-covered target surfaces. ${ }^{7-9}$ The studies suggest the order of GPa for the plume pressure in an initial stage of the ablation. ${ }^{7}$ On the other hand, optical emission spectra ${ }^{10}$ and emission imaging ${ }^{11}$ can also be used for the study of the plume properties. Because of a high density in this region, however, the emission spectra suffer from strong selfabsorption. We have analyzed quantitatively the selfabsorption effect upon the line emission of $\mathrm{Al}$ atoms in the plume produced by the laser ablation of $\mathrm{Al}$ metal in water. ${ }^{12}$ It is also known that the ablation plume, even in a gas atmosphere, shows clear self-absorption in the spectral profile of a line emission. ${ }^{16}$ The density of the $\mathrm{Al}$ atoms in a laser ablation plume can be obtained by analyzing the line profile of the emission spectra. ${ }^{16,17}$

The temperature of the ablation plume produced in water by irradiating a graphite target submerged in water has been determined as a function of time by the analysis of the $\mathrm{C}_{2}$ Swan band. ${ }^{13,14}$ The Swan band shows clearly resolved rotational lines, suggesting that the $\mathrm{C}_{2}$ molecules are rotating, and also that the plume is in a gaseous phase with a very high density of the ablated species. ${ }^{14}$ However, the best-fit

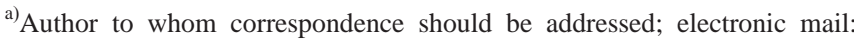
t-sakka@iae.kyoto-u.ac.jp
}

spectra had an inevitable deviation from the experimental spectra, probably because the self-absorption effect had not been taken into account. ${ }^{14}$

In the present work, we report that the spectral profile of the $\mathrm{C}_{2}$ Swan band emission can be reproduced with further accuracy by taking the self-absorption effects into account and adjusting the optical density parameter. The best-fit optical density parameter was obtained by fitting the whole profile of the $(0,0)$ band of the Swan system. The optical density at the band head was obtained as a function of time. The results give insight into the density of the $\mathrm{C}_{2}$ molecules in the plume, and hence, into the confinement effects of the plume in liquids.

\section{CALCULATION}

The observed spectra analyzed in the present work were taken from our previous work, ${ }^{14}$ where the emission spectra of the $(0,0)$ band of the $\mathrm{C}_{2}$ Swan system were measured at various delay times from the ablation laser. The Nd:YAG laser pulse with the wavelength of $1064 \mathrm{~nm}$, the pulse width of $20 \mathrm{~ns}$, and the fluence of $30 \mathrm{~mJ}$ was focused down onto the target surface by a $100 \mathrm{~mm}$ focal length lens. The consecutive five irradiation events for each delay time were averaged and compared with theoretical spectra. The spectral resolution of the data was $0.03 \mathrm{~nm}$.

Calculation of the theoretical spectra is based on the Hönl-London factors given in the literature ${ }^{18}$ and the Boltzmann factor. The details of the calculation for the lowoptical-density limit, where self-absorption effects are not taken into account, are given in the previous work. ${ }^{14}$ Rotational temperature and rotational line width were obtained as best-fit parameters by comparing theoretical spectra with the experimental ones.

Self-absorption effects were introduced in the calculation on the basis of one-dimensional radiative transfer with assuming a homogeneous media for the emission and absorption. The intensity of the emission from a homogeneous media is given as follows: ${ }^{16}$ 


$$
I=\frac{\varepsilon}{\kappa}[1-\exp (-\kappa L)],
$$

where $\varepsilon$ and $\kappa$ are the emission and absorption coefficients, respectively, and $L$ is the geometrical thickness of the emission region. For the limit of low density where the absorption is negligible, and hence, $\kappa L$ tends to be zero, $I_{\kappa=0}=\varepsilon L$. Therefore, from Eq. (1),

$$
I=I_{\kappa=0} \frac{1}{\kappa L}[1-\exp (-\kappa L)] .
$$

By substituting $\kappa=\alpha \varepsilon$ and using $I_{\kappa=0}=\varepsilon L$ we obtain

$$
I=\frac{1}{\alpha}\left[1-\exp \left(-\alpha I_{\kappa=0}\right)\right] .
$$

Note that the parameter $\alpha$ depends only on the population densities of the upper and lower levels involved in the transition, and does not depend on the wavelength, although $\kappa$ and $\varepsilon$ are strong functions of the wavelength. Also, note that $I_{\kappa=0}$ is a theoretical spectrum calculated without considering self-absorption effects. Therefore, Eq. (3) means that the spectra with self-absorption effects taken into account can be obtained by considering one more parameter $\alpha$.

In the actual fitting process we first obtained $I_{\kappa=0}$ by fitting the band tail region, where the optical thickness is expected to be small. ${ }^{14}$ Then, the whole spectral profile was fitted to Eq. (3) without further adjustment of $I_{\kappa=0}$. This process gives a best-fit $\alpha$ parameter. The optical density at the band head was calculated by $\kappa^{\mathrm{BH}} L=\alpha \varepsilon^{\mathrm{BH}} L=\alpha I_{\kappa=0}^{\mathrm{BH}}$, where superscript "BH" means "band head."

\section{RESULTS}

In Fig. 1, observed spectra in a later time of the emission and their best-fit spectra, including self-absorption effects, are shown. Figure 1(a) shows the spectrum obtained in air at a delay time of $1200 \mathrm{~ns}$, while Fig. 1(b) gives the spectrum obtained by the irradiation of the target immersed in water at a delay time of $1000 \mathrm{~ns}$. The best-fit spectra given by the broken line reproduced almost exactly the experimental spectra for both cases. The observed emission spectra from the water-confined plume at early delay times as well as their best-fit spectra are given in Fig. 2. The band-head intensity is comparable to the band-tail intensity at very early delay times, e.g., the delay time of $50 \mathrm{~ns}$. Such spectra can also be reproduced quite well by theoretical spectra.

In Fig. 3, the optical density at the band head $\kappa^{\mathrm{BH}} L$ is plotted as a function of time. Both in water and in air, the optical density decreases with time. The optical densities of the plumes in water tend to give larger values, especially in the early time range. The optical densities of the plumes in air were much smaller than those obtained in water, but still give values around the unity, while it should approach zero at the limit of the low density of $\mathrm{C}_{2}$ molecules.

\section{DISCUSSION}

As can be seen in Figs. 1(b) and 2, the fit of the theoretical spectra to the observed one is excellent. This should be compared with the results obtained without considering the

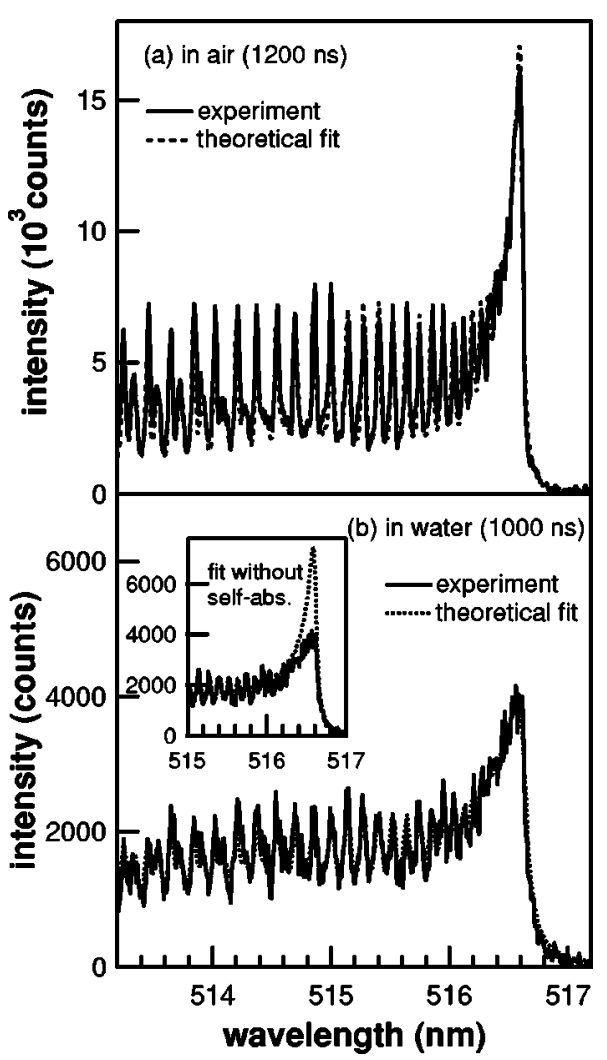

FIG. 1. Examples of the best-fit spectra for the $(0,0)$ band of the $\mathrm{C}_{2}$ Swan system; (a) in air at a delay time of $1200 \mathrm{~ns}$, (b) in water at $1000 \mathrm{~ns}$. The experimental spectra are given by a solid line. Also, the result of the fitting without considering the self-absorption effect is given by the dotted line in the inset of (b).

self-absorption effects, shown in the inset of Fig. 1(b); the band head intensity is far from a "good fit," although the fit in the band-tail region is very good. The excellent fit in Figs. 1(b) and 2 supports the interpretation that the self-absorption strongly affects the band profile.

The plot of the optical density in Fig. 3 shows some scattering of the data points in the case of the plume in water. This is because the exponential factor in Eq. (3), i.e., $\left[1-\exp \left(-\alpha I_{\kappa=0}\right)\right]$, becomes rather insensitive to the parameter $\alpha$, when $\alpha I_{\kappa=0}$ becomes large, and therefore, a small deviation in the spectral profile results in a big change in the fitting parameter $\alpha$. In Fig. 2, we actually see that at the early delay times, the intensity of the band head is comparable to that of the band tail. This effect is prominent for the plume in water at the delay time of 50 ns (open square in Fig. 3). In spite of the scattering of the data, it seems obvious that the optical density of the plume in water is much higher than that in air. Furthermore, we think that the slight decrease in the optical density observed both for the plumes in water and in air in this time range is meaningful.

The optical density obtained as a function of delay time gives some insight into the time evolution of the ablation plume produced in liquids. The large optical density of the plume in water supports a strong confinement of the ablation plume in liquids. It has been reported that the confinement is so strong as to give a pressure over $1 \mathrm{GPa}$ in this region. ${ }^{7,11}$ The imaging of the optical emission from the plume shows that the size of the plume is ca. $0.1 \mathrm{~mm}$ in its diameter of the 


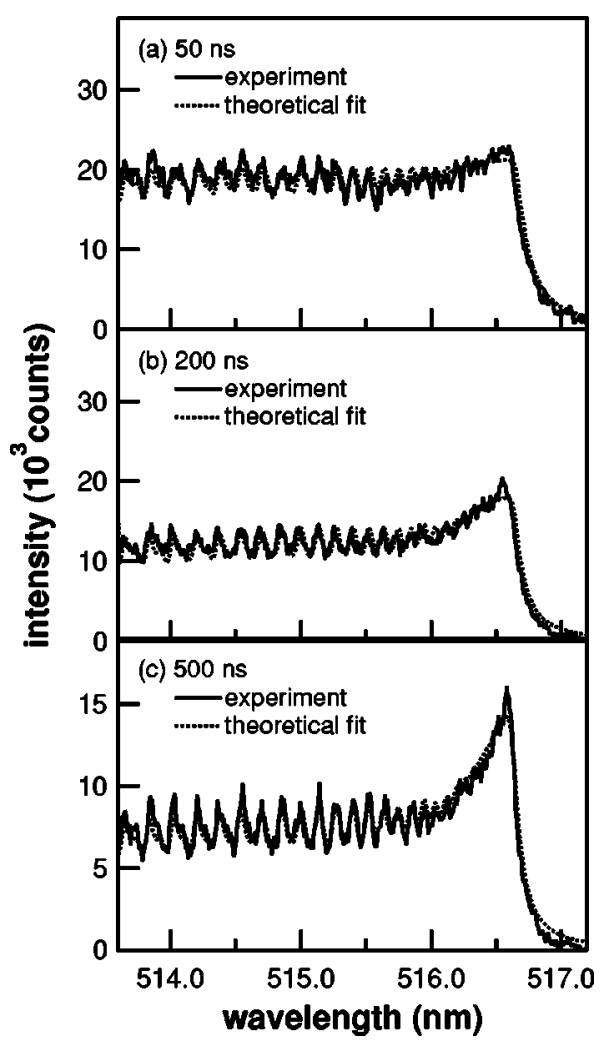

FIG. 2. Example of the observed and best-fit spectra at relatively early delay times. The delay time of each spectrum is (a) $50 \mathrm{~ns}$, (b) $200 \mathrm{~ns}$, and (c) 500 ns.

half-maxima contour. ${ }^{11}$ This is in contrast to the ablation plume in air, where the plume size ranges in the order of $\mathrm{mm}$.

Since the absorption coefficient $\kappa$ is proportional to the difference between the population densities of the two levels involved in the transition, it decreases by $L^{-3}$ when the plume expands in three dimensions. This means that the optical density, $\kappa L$, decreases roughly by $L^{-2}$ upon expansion of the plume. By assuming the total amount of $\mathrm{C}_{2}$ in the plume to be constant, the optical density should be low for the plume having expanded. The difference between the ablation in air and in water is a direct consequence of the ex-

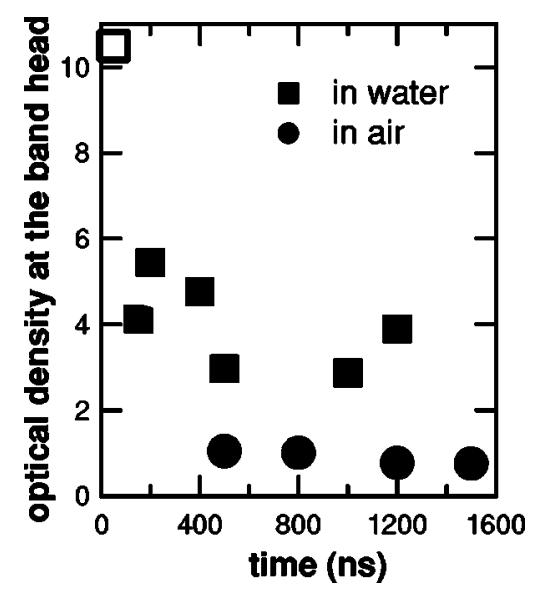

FIG. 3. Optical densities of the plume at the wavelength of the band head as a function of the delay time. They were obtained by fitting to the theoretical spectra with self-absorption effects being taken into account. pansion behavior of the plume, i.e., the plume having undergone a relatively free expansion in air gives lower optical densities while the confined plume in water gives high optical densities. The results in Fig. 3 clearly reflect the fact that the plume expansion in water is confined strongly to give high optical density.

Also, the slight decrease of the optical density observed for both in water and in air at longer delay times can be interpreted by the plume expansion. However, the decrease in the optical density is rather slow. Our recent observation of the shadow graph of the plume, which measures the size of the plume after the extinction of the optical emission, suggests such an expansion rate that the plume diameter increases to $1 \mathrm{~mm}$ by ca. $20 \mu \mathrm{s}$ after the irradiation of the ablation laser. This corresponds to a $0.05 \mathrm{~mm}$ increase in the plume diameter within the time range of the present work. Since the diameter of the emission region has been measured to be ca. $0.1 \mathrm{~mm}$ immediately after the ablation laser (ca. 30 ns of the delay time), ${ }^{11}$ the expansion is not necessarily negligible. Although the emission imaging of the plume shows that the size of the emission region is almost constant, ${ }^{11}$ we think that a slight expansion of the plume causes a small change in the density of $\mathrm{C}_{2}$ molecules, and hence, to the optical density of the plume. However, another interpretation, as based on the decrease in the density of the $\mathrm{C}_{2}$ molecules due to some reactions of $\mathrm{C}_{2}$, seems to be possible. Further study is required for the clarification of the chemical and physical behavior of the ablation species in the plume produced in liquids.

As shown above the present analysis is quantitative in the time range, where the optical density is not too high, and also where clear emission is observed. The former condition prevents us from obtaining quantitative information in the very early stage of the laser ablation plume in water. The theory of the shock wave predicts that the pressure maximum almost coincides with the peak of the irradiation laser intensity profile, ${ }^{19}$ i.e., several nanoseconds to 10 for the nanosecond laser pulse irradiation. This means that the initial strong expansion of the plume against water stops at this time range. This suggests that it is hard to study the plume dynamics in very initial stage by the present method or by any detecting system using an intensified charge coupled device (ICCD) detecting systems. We think that the pump-probetype experiment using the ablation and probe pulse width of $1 \mathrm{~ns}$ or shorter would be helpful for the clarification of the plume behavior in this time range.

\section{CONCLUSION}

The emission spectra of the $(0,0)$ band in the Swan system of the $\mathrm{C}_{2}$ molecules in the laser ablation plume of the graphite target in water were studied. The spectra have been excellently reproduced by introducing the self-absorption effect in the model calculation in addition to the low-densitylimit calculations. Optical density of the plume, obtained as a best-fit parameter, shows that it is higher for the plumes pro- 
duced in water than in air, and shows a slight decrease with increasing delay time. These results are interpreted in terms of the expansion of the plume.

${ }^{1}$ S. B. Ogale, P. P. Patil, D. M. Phase, Y. V. Bhandarker, S. K. Kulkarni, S. A. Kulkarni, V. G. Bhide, and S. Guha, Phys. Rev. B 36, 8237 (1987).

${ }^{2}$ J. Singh, M. Vellaikai, and J. Narayan, J. Appl. Phys. 73, 4351 (1993).

${ }^{3}$ C. H. Liang, T. Sasaki, Y. Shimizu, and N. Koshizaki, Chem. Phys. Lett. 389, 58 (2004).

${ }^{4}$ J. B. Wang and G. W. Yang, J. Phys.: Condens. Matter 11, 7089 (1999).

${ }^{5}$ C. H. Liu, W. Peng, and L. M. Sheng, Carbon 39, 137 (2001).

${ }^{6}$ G. Compagnini, A. A. Scalisi, and O. Puglisi, ChemPhysChem 4, 2787 (2002).

${ }^{7}$ L. Berthe, R. Fabbro, P. Peyre, L. Tollier, and E. Bartnicki, J. Appl. Phys. 82, 2826 (1997).

${ }^{8}$ S. Zhu, Y. F. Lu, and M. H. Hong, Appl. Phys. Lett. 79, 1396 (2001).
${ }^{9}$ S. Zhu, Y. F. Lu, M. H. Hong, and X. Y. Chen, J. Appl. Phys. 89, 2400 (2001).

${ }^{10}$ T. Sakka, S. Iwanaga, Y. H. Ogata, A. Matsunawa, and T. Takemoto, J. Chem. Phys. 112, 8645 (2000).

${ }^{11}$ K. Saito, K. Takatani, T. Sakka, and Y. H. Ogata, Appl. Surf. Sci. 197, 56 (2002).

${ }^{12}$ T. Sakka, K. Takatani, and Y. H. Ogata, J. Phys. D 35, 65 (2002).

${ }^{13}$ T. Sakka, K. Saito, and Y. H. Ogata, Appl. Surf. Sci. 197, 246 (2002).

${ }^{14}$ K. Saito, T. Sakka, and Y. H. Ogata, J. Appl. Phys. 94, 5530 (2003).

${ }^{15}$ V. A. Ageev, A. Fbokhonov, V. V. Zhukovskii, and A. A. Yankovskii, J. Appl. Spectrosc. 64, 683 (1997).

${ }^{16}$ T. Sakka, T. Nakajima, and Y. H. Ogata, J. Appl. Phys. 92, 2296 (2002).

${ }^{17}$ H. Furusawa, T. Sakka, and Y. H. Ogata, J. Appl. Phys. 96, 975 (2004).

${ }^{18}$ I. Kovacs, Rotational Structure in the Spectra of Diatomic Molecules (Akadémiai Kiadó, Budapest, 1969).

${ }^{19}$ D. Devaux, R. Fabbro, L. Tollier, and E. Bartnicki, J. Appl. Phys. 74, 2268 (1993). 